\title{
Social Ranking Manipulability for the CP-Majority, Banzhaf and Lexicographic Excellence Solutions*
}

\author{
Tahar Allouche $^{1}$, Bruno Escoffier ${ }^{2,3}$, Stefano Moretti ${ }^{4}$ and Meltem Öztürk ${ }^{4 \dagger}$ \\ ${ }^{1}$ ENSTA-Paris, Institut Polytechnique de Paris, France \\ ${ }^{2}$ Sorbonne Université, CNRS, LIP6, F-75005 Paris, France \\ ${ }^{3}$ Institut Universitaire de France, France \\ ${ }^{4}$ Université Paris Dauphine, PSL Research University, CNRS, Lamsade, 75016 Paris, France \\ tahar.allouche@ensta-paris.fr, Bruno.Escoffier@lip6.fr, \{stefano.moretti, meltem.ozturk\}@dauphine.fr
}

\begin{abstract}
We investigate the issue of manipulability for social ranking rules, where the goal is to rank individuals given the ranking of coalitions formed by them and each individual prefers to reach the highest positions in the social ranking. This problem lies at the intersection of computational social choice and the algorithmic theory of power indices. Different social ranking rules have been recently proposed and studied from an axiomatic point of view. In this paper, we focus on rules representing three classical approaches in social choice theory: the marginal contribution approach, the lexicographic approach and the (ceteris paribus) majority one. We first consider some particular members of these families analysing their resistance to a malicious behaviour of individuals. Then, we analyze the computational complexity of manipulation, and complete our theoretical results with simulations in order to analyse the manipulation frequencies and to assess the effects of manipulations.
\end{abstract}

\section{Introduction}

In decision making and social choice theory, a number of studies are devoted to ranking individuals based on the performance of the coalitions formed by them. For instance, given values on coalitions of individuals, power indices map these values of coalitions on values of individuals. The seminal works of Shapley [1953] and Banzhaf III [1964] paved the way of a whole research domain and a related literature with many issues, including axiomatization [Laruelle and Valenciano, 2001; Holler and Packel, 1983], applications [Bilbao et al., 2002; Moretti and Patrone, 2008], algorithmic analysis [Matsui and Matsui, 2000] and computational complexity [Deng and Papadimitriou, 1994; Bachrach and Rosenschein, 2009; Faliszewski and Hemaspaandra, 2009]. The nonmanipulability (or strategy proofness) is another fundamental issue. In social choice, since the seminal theorems of Gibbard and Satterthwaite ([Gibbard, 1973] and [Satterthwaite,

\footnotetext{
${ }^{*}$ We are grateful to anonymous reviewers for their critical and helpful comments.

${ }^{\dagger}$ Contact author.
}

1975]), we know that every interesting social choice function is manipulable by misrepresentation of preferences. The manipulability is also analysed for power indices. We quote in particular the literature on the paradoxical behaviour of power indices under the modification of some elements of the game, like the number of players or the size of coalitions [Felsenthal et al., 1998; Laruelle and Valenciano, 2005], or the study of manipulation in weighted voting games [Aziz et al., 2011; Zuckerman et al., 2012]. In these models, players are analyzed from a strategic perspective to establish under which conditions they can increase their power adopting malicious behaviors like, for example, splitting or merging.

Power indices (and other indices of individual productivity based on the evaluation of revenues generated by teams [Flores-Szwagrzak and Treibich, 2020]) require a numerical evaluation of coalitions of individuals. Following classical situations in social choice where ordinal data are provided (for instance, voting theory), several articles address the question of defining ordinal notions of power indices when we only have ordinal information over coalitions. This has been formalized as the social ranking from coalitions (SRC) problem, where the objective is to evaluate the "influence" of individuals involved in a collective decision process like an electoral system, a parliament, a governing council, a management board, etc. ([Moretti, 2015; Moretti and Öztürk, 2017]). Basically, an SRC problem consists of a finite set $N$ of individuals and a binary relation $\succeq$ over some subsets (hereafter called coalitions) of $N$; the binary relation $\succeq$ is called power relation and represents the relative power of coalitions in a decision process. A solution or rule for an SRC problem is a "suitable" method aimed to convert the information contained in a power relation $\succeq$ into a ranking over the single elements of $N$ representing their overall individual power. Several solutions for SRC problems have been proposed in the literature. For instance, in the work by Haret et al. [2018] (and the one by Fayard and Öztürk [2018]) two individuals are compared using information from subsets ranking them under a ceteris paribus interpretation. Bernardi et al. [2019] axiomatically characterize a solution based on the idea that the most influential individuals are those appearing more frequently in the highest positions of the power relation. A rule based on the idea of ordinal marginal contribution has been recently introduced in the paper by Khani et al. [2019]. 
Following these lines of research, in this article we are interested in the analysis of the strategic manipulation of SRC rules, in the sense that an individual may be interested in behaving maliciously within one or more teams (weakening their group's effectiveness) in order to obtain a better position in the individual ranking. The notion of manipulability for SRC considered in this paper assumes that an individual can only weaken the ranking of teams to which she belongs. In other words, an individual $i$ cannot affect the performances of teams not containing $i$ and, in addition, cannot improve the position of a coalition already containing $i$.

Example 1. Consider a manager who must decide how to allocate two bonuses over three employees (denoted by 1,2 and 3). Suppose that she can only compare the performance of teams in an ordinal way: $\{1,2,3\} \succ\{1,3\} \succ\{1\} \succ$ $\{1,2\} \succ\{2,3\} \succ\{2\} \succ\{3\} \sim \emptyset^{1}$. Suppose the manager wants to keep into account the attitude of employees to cooperate. So, an option is to count the number of (ordinal) positive and negative marginal contributions provided by each employee to all possible coalitions, i.e. 1 contributes positively to four teams (i.e., $\{1,2,3\} \succ\{2,3\},\{1,3\} \succ\{3\}$, $\{1,2\} \succ\{2\}$ and $\{1\} \succ \emptyset), 3$ contributes positively to three coalitions while 2 also contributes positively to three coalitions, but negatively to coalition $\{1,2\}$. Therefore the manager would end up to award players 1 and 3. Such a rule could push individual 2 to behave strategically and to undermine the cooperation within coalition $\{2,3\}$. So the new ranking being $\{1,2,3\} \succ^{\prime}\{1,3\} \succ^{\prime}\{1\} \succ^{\prime}\{1,2\} \succ^{\prime}$ $\{2\} \succ^{\prime}\{2,3\} \succ^{\prime}\{3\} \sim^{\prime} \emptyset$, individuals 1 and 2 should now get the bonus.

To our knowledge, this article is the first one which investigates the manipulation for SRC rules. As in social choice, the manipulability is an important issue in many real-world coalitional frameworks. For instance, within a parliament, small political parties are often able to blackmail a majority coalition by threatening to withdraw from the coalition or to run a candidate of their own [Ferrara, 2004]. In international organizations, the capacity to block the proposals of a group ensures a great visibility of the blocking state's positions. In any organizational context, like a business company or an academic institution, any perceived disparity about the merits of a team's members (e.g., over-remuneration of a leader) may engender internal competition, or even the sabotage of the team by some of it's members. As a sensible example of this kind of behaviors, consider the situation following the Italian general election of March 2018, where no political party got an absolute majority. After 3 months of negotiation, a coalition government was finally formed by the two parties casting the highest number of votes, precisely, the Five Star Movement and the League. However, such a government ended one year after, in August 2019, when the League withdrew its support to the coalition government. As a consequence, the League substantially increased its position in the opinion polls done immediately after the government crisis [Chiaramonte et al., 2020].

The article is organized as follows. We present basic notions and notations in Section 2. In Section 3, we intro-

\footnotetext{
${ }^{1} \succ:$ strict preference, $\sim$ : indifference.
}

duce a formal definition of manipulability for an SRC rule and provide theoretical results on four social ranking rules: Copeland-like, Kramer-Simpson-like, Lexicographic Excellence [Bernardi et al., 2019] and Ordinal Banzhaf [Khani et al., 2019]. These social ranking rules display a wide variety of characteristics. The two first ones are based on ceteris paribus comparisons and use the majority principle in a different way. Copeland-like solution is a kind of flow analysis of majority graphs, whereas Kramer-Simpson is a minmax score. Ordinal Banzhaf rule is based on a marginal contribution principle. Lexicographic Excellence (lexcel) considers only information from the best ranked coalitions. We show that only lexcel is not manipulable. In Section 4, we analyse the computational complexity of manipulating each of the three manipulable social ranking rules, and prove that for each of them determining whether an individual can manipulate or not is an NP-hard problem. In Section 5, we present some simulation results on manipulable social ranking rules showing the manipulation frequencies and their vulnerability against the manipulation. Section 6 concludes the article.

\section{Preliminaries}

Let $N=\{1, \ldots, n\}$ be a finite set of elements called individuals and let $R \subseteq N \times N$ be a binary relation on $N$. A preorder is a reflexive and transitive binary relation. A preorder that is total is called total preorder. An antisymmetric ${ }^{2}$ total preorder is called linear order. We denote by $\mathcal{T}(N)$ the set of all total preorders on $N$ and by $2^{N}$ the powerset of $N$, i.e. the set of all subsets (also called, coalitions) of $N$. Let $\mathcal{P} \subseteq 2^{N}$ be a non-empty collection of subsets of $N$. A power relation on $\mathcal{P}$ is a total preorder $\succeq \subseteq \mathcal{P} \times \mathcal{P}$. We denote by $\mathcal{T}(\mathcal{P})$ the family of all power relations on every non-empty collection $\mathcal{P} \subseteq 2^{N}$. Given a power relation $\succeq \in \mathcal{T}(\mathcal{P})$ on $\mathcal{P} \subseteq 2^{N}$, we denote by $\sim$ its symmetric part (i.e. $S \sim T$ if $S \succeq T$ and $T \succeq S$ ) and by $\succ$ its asymmetric part (i.e. $S \succeq T$ and not $T \succeq S$ ). So, for each pair of subsets $S, T \in \mathcal{P}, S \succ T$ means that $S$ is strictly stronger than $T$, whereas $S \sim T$ means that $S$ and $T$ are indifferent.

Let $\succeq \in \mathcal{T}(\mathcal{P})$ be of the form $S_{1} \succeq S_{2} \succeq S_{3} \succeq \cdots \succeq$ $S_{|\mathcal{P}|}$. The quotient order of $\succeq$ is denoted as $\Sigma_{1} \succ \Sigma_{2} \succ$ $\Sigma_{3} \succ \cdots \succ \Sigma_{m}$ in which the subsets $S_{j}$ are grouped in the equivalence classes $\Sigma_{k}$ generated by the symmetric part of $\succeq$. This means that all the sets in $\Sigma_{1}$ are indifferent to $S_{1}$ and are strictly better than the sets in $\Sigma_{2}$ and so on. So, $\Sigma_{i}=S_{i}$ for any $i=1, \ldots,|\mathcal{P}|$ if and only if $\succeq$ is a linear order.

A social ranking solution or solution on $N$, is a function $R: \mathcal{T}(\mathcal{P}) \longrightarrow \mathcal{T}(N)$ associating to each power relation $\succeq \in$ $\mathcal{T}(\mathcal{P})$ a total preorder $R(\succeq)$ (or $R^{\succeq}$ ) over the elements of $N$. By this definition, the notion $i R^{\succeq} j$ means that applying the social ranking solution to the power relation $\succeq$ gives the result that $i$ is ranked higher than or equal to $j$. We denote by $I^{\succeq}$ the symmetric part of $R \succeq$, and by $P^{\succeq}$ its asymmetric part. The social score $p_{i}\left(R^{\succeq}\right)$ of individual $i \in N$ in $R^{\succeq}$ is defined as the number of individuals in $N \backslash\{i\}$ that are ranked lower than $i$ minus the number of individuals in $N \backslash\{i\}$ that are ranked higher than $i$, that is

$p_{i}\left(R^{\succeq}\right)=\left|\left\{j \in N \backslash\{i\}: i R^{\succeq} j\right\}\right|-\left|\left\{j \in N \backslash\{i\}: j R^{\succeq} i\right\}\right|$.
${ }^{2} \forall i, j \in N, i R j$ and $j R i \Rightarrow i=j$. 


\section{Manipulability}

In this paper we focus on a particular notion of manipulation, intended as the "unlimited" capacity of individuals to undermine the position of coalitions to which they belong in a power relation $\succeq$ on $\mathcal{P} \subseteq 2^{N}$.

Definition 1. Let $\succeq \in \mathcal{T}(\mathcal{P})$ on $\mathcal{P}$ be a power relation with the associated quotient order $\succ$ :

$$
\Sigma_{1} \succ \Sigma_{2} \succ \cdots \succ \Sigma_{j} \succ \cdots \succ \Sigma_{m} .
$$

Let $i$ be an individual, and $\mathcal{C} \subseteq \mathcal{P}$ be a collection of coalitions in $\mathcal{P}$ all containing $i$. For all $S \in \mathcal{C}$, let $j(S) \in\{1, \ldots, m\}$ be such that $S \in \Sigma_{j(S)}{ }^{3}$.

A manipulation of $\succeq$ by individual $i$ via collection $\mathcal{C}$ is another power relation $\succeq^{\mathcal{C}}$ on $\mathcal{P}$ with $\succeq^{\mathcal{C}} \neq \succeq$ and with the associated quotient order $\succ^{\mathcal{C}}$ such that the following two conditions hold:

i) $\Sigma_{1} \backslash \mathcal{C} \succ^{\mathcal{C}} \Sigma_{2} \backslash \mathcal{C} \succ^{\mathcal{C}} \cdots \succ^{\mathcal{C}} \Sigma_{j} \backslash \mathcal{C} \succ^{\mathcal{C}} \cdots \succ^{\mathcal{C}} \Sigma_{m} \backslash \mathcal{C}$;

ii) $T \succ^{\mathcal{C}} S$ for all $S \in \mathcal{C}$ and $T \in \bigcup_{i=1, \ldots, j(S)} \Sigma_{i} \backslash \mathcal{C}$.

A social ranking $\mathcal{R}$ is manipulable by $i$ on a power relation $\succeq$ on $\mathcal{P}$ if there exists a collection of coalitions $\mathcal{C} \subseteq \mathcal{P}$ containing $i$, a manipulation $\succeq^{\mathcal{C}}$ of $\succeq$ by $i$ via $\mathcal{C}$ such that

$$
p_{i}\left(R^{\succeq^{c}}\right)>p_{i}\left(R^{\succeq}\right) .
$$

[recall that $\left.\left.p_{i}\left(R^{\succeq}\right)\right)=\left|\left\{j, i R^{\succeq} j\right\}\right|-\left|\left\{j, j R^{\succeq} i\right\}\right|.\right]$

A social ranking solution $\mathcal{R}$ is manipulable on a power relation $\succeq$ if it is manipulable by some individual $i$.

Condition (ii) says that $\succeq^{\mathcal{C}}$ is obtained from $\succeq$ moving each coalition $S \in \mathcal{C}$ from the equivalence class to which it belongs in $\succeq$, to a strictly lower equivalence class (that can also be a new singleton equivalence class containing only $S$ in $\succeq^{\mathcal{C}}$ ), while the relation among all the other coalitions not in $\mathcal{C}$ is maintained as in $\succeq$ (condition $(i)$ ). The family of all manipulations of $\succeq$ via collection $\mathcal{C}$ is denoted by $\mathcal{M}_{\mathcal{C}}(\succeq)$.

Example 2. Consider the power relation $\succeq$ such that $t^{4} 23 \succ$ $(123 \sim 12) \succ 13$ (hence, $\Sigma_{1}=\{23\}, \Sigma_{2}=\{123,12\}, \Sigma_{3}=$ $\{13\})$. Imagine that individual 1 wants to manipulate by deteriorating the positions of coalitions in $\mathcal{C}=\{12,13\}$. Condition i) of Definition 1 imposes to maintain $23 \succ^{\mathcal{C}} 123$, condition ii) imposes $23 \succ^{\mathcal{C}} 12,23 \succ^{\mathcal{C}} 13,123 \succ^{\mathcal{C}} 12$ and $123 \succ^{\mathcal{C}} 13$. Hence, the family of all possible manipulations of $\succeq$ by 1 via collection $\mathcal{C}$ is $\mathcal{M}_{\mathcal{C}}(\succeq)=\left\{\succeq_{a}^{\mathcal{C}}, \succeq_{b}^{\mathcal{C}}, \succeq_{c}^{\mathcal{C}}\right\}$, with $23 \succ_{a}^{\mathcal{C}} 123 \succ_{a}^{\mathcal{C}} 12 \succ_{a}^{\mathcal{C}} 13,23 \succ_{b}^{\mathcal{C}} 123 \succ_{b}^{\mathcal{C}} 13 \succ_{b}^{\mathcal{C}} 12$ and $23 \succ_{c}^{\mathcal{C}} 123 \succ_{c}^{\mathcal{C}} 12 \sim_{c}^{\mathcal{C}} 13$.

We will now analyse the manipulability of different social ranking rules.

\subsection{Copeland-like and Kramer-Simpson-like Rules}

Copeland-like and Kramer-Simpson (KS)-like rules are both based on Ceteris Paribus-majority relation, where individuals $i$ and $j$ are ranked according to their relative success over comparisons of coalitions of the type $S \cup i$ vs. $S \cup j$ (CPcomparisons), more precisely:

\footnotetext{
${ }^{3} j(S)$ represents the rank of the equivalence class to which $S$ belongs in the initial power relation $\succeq$.

${ }^{4}$ To avoid cumbersome notations later, sets will be written for short without commas and parentheses, e.g., 123 instead of $\{1,2,3\}$, and $S \cup i$ instead of $S \cup\{i\}$.
}

Definition 2 (CP-Majority [Haret et al., 2018]). Let $\succeq \in$ $\mathcal{T}(\mathcal{P})$. The Ceteris Paribus (CP-) majority relation is the binary relation $R_{\bar{C} P}^{\succ} \subseteq N \times N$ such that for all $i, j \in N$ :

$$
i R_{\bar{C} P}^{\succeq} j \Leftrightarrow d_{i j}(\succeq) \geq d_{j i}(\succeq),
$$

where $d_{i j}(\succeq)$ represents the cardinality of the set $D_{i j}(\succeq)$, the set of all coalitions $S \in 2^{N-\{i, j\}}$ for which $S \cup i \succeq S \cup j^{5}$.

Example 3. Consider: $(123 \sim 12 \sim 3 \sim 1) \succ(2 \sim 23) \succ$ 13. Then, we obtain $1 I_{\bar{C} P}^{\succ} 2,1 P_{\bar{C} P}^{\succ} 3,2 I_{\bar{C} P}^{\succ} 3$. For instance, $1 I_{\bar{C} P}^{\succ} 2: D_{12}(\succeq)=\{\emptyset\}(1 \succ 2) ; D_{21}(\succeq)=\{3\}(23 \succ 13)$.

The CP-Majority relation has a major drawback: it can generate cycles within the individual ranking (except under some particular domain restrictions, as suggested in [Haret et $a l ., 2018])$. For this reason, we investigate the manipulability of two transitive solutions derived from the CP-Majority, which are inspired, respectively, by the Copeland [Copeland, 1951] and Kramer-Simpson [Simpson, 1969] [Kramer, 1977] voting schemes. These two rules are known to be Condorcet coherent, meaning that when a Condorcet winner (a candidate beating all the other candidates by the majority rule) exists, it is chosen by them. Interestingly, while it can be easily proved that $\mathrm{CP}$-majority relation is not manipulable, Copeland like and KS like solutions are manipulable.

\section{Copeland-like Method}

Strongly inspired by the Copeland score of social choice theory, we define Copeland-like solution based on the net flow of CP-majority graph. According to the Copeland solution, individuals are ordered according to the number of pairwise winning comparisons, minus the one of pairwise losing comparisons, over the set of all CP-comparisons.

Definition 3 (Copeland-like solution). Let $\succeq \in \mathcal{T}(\mathcal{P})$. The Copeland-like relation is the binary relation $R_{\bar{C} \text { op }}^{\succ} \subseteq N \times N$ such that for all $i, j \in N$ :

$$
\begin{gathered}
i R_{\overline{C o p}}^{\succ} j \Leftrightarrow S \text { core }_{\text {cop }}^{\succ}(i) \geq S \text { core } e_{\text {cop }}^{\succ}(j) . \\
\text { where Score } e_{\text {cop }}^{\succ}(i)=p_{i}\left(R_{\bar{C} P}^{\succ}\right)= \\
\left|\left\{j \in N \backslash\{i\}: i R_{\bar{C} P}^{\succ} j\right\}\right|-\left|\left\{j \in N \backslash\{i\}: j R_{\bar{C} P}^{\succ} i\right\}\right| .
\end{gathered}
$$

\section{Theorem 1. The Copeland-like solution is manipulable.}

Proof. See Example 4 for an instance of manipulation.

Example 4. Consider $\succeq$ of Example 3. Then,

$$
\text { Score } e_{\text {cop }}^{\succ}(1)=1, \text { Score }_{\text {cop }}^{\succ}(2)=0, \text { Score } e_{\text {cop }}^{\succeq}(3)=-1 \text {. }
$$

Hence, the Copeland-like relation is: $1 P_{\overline{C o p}}^{\succ} 2 P_{\bar{C} o p}^{\succ} 3$. Now imagine that 3 deteriorates the performance of $23(\mathcal{C}=\{23\})$

$$
\left(123 \sim^{\mathcal{C}} 12 \sim^{\mathcal{C}} 3 \sim^{\mathcal{C}} 1\right) \succ^{\mathcal{C}} 2 \succ^{\mathcal{C}} 13 \succ^{\mathcal{C}} 23 .
$$

Now we have: Score $e_{\text {cop }}^{\succ^{c}}(1)=2$, Score $e_{\text {cop }}^{\succ^{c}}(2)=-1$, Score $e_{\text {cop }}^{\succ^{c}}(3)=-1$. So, now, 3 shares the second position with 2 .

\footnotetext{
${ }^{5}$ Note that $S$ can be $\emptyset$
} 


\section{Kramer-Simpson-like Method}

Strongly inspired by the Kramer-Simpson method of social choice theory (Minmax), individuals are ranked inversely to their greatest pairwise defeat over all possible $\mathrm{CP}$ comparisons.

Definition 4 (Kramer-Simpson-like solution). Let $\succeq \in \mathcal{T}(\mathcal{P})$. The KS-like relation (KS relation) is the binary relation $R_{\bar{K} S}^{\succ} \subseteq N \times N$ such that for all $i, j \in N$ :

$$
i R_{\bar{K} S}^{\succ} j \Leftrightarrow \operatorname{Score}_{\bar{K} S}^{\succ}(i) \leq \operatorname{Score}_{\bar{K} S}^{\succ}(j),
$$

where $\operatorname{Score} e_{K S}^{\succeq}(i)=\max _{j \in N}\left(d_{j i}(\succeq)\right)$

Theorem 2. The Kramer-Simpson (KS)-like solution is manipulable.

Proof. See Example 5 for an instance of manipulation.

Example 5. Consider $\succeq: 2 \succ(1 \sim 3) \succ 12 \succ$ $(13 \sim 23) \succ \emptyset \succ 123$. Then, $S \operatorname{scor} e_{K S}^{\succ}(1)=$ 1, Score $e_{\bar{K}}^{\succ}(2)=0, S$ core $e_{\bar{K}}^{\succ}(3)=2$. Hence, KS-like solution is: $2 P_{\bar{K} S}^{\succ} 1 P_{\bar{K} S}^{\succ}$. Now consider the following manipulation operated by 1 on $\mathcal{C}=\{12\}$ :

$$
2 \succ^{\mathcal{C}}\left(1 \sim^{\mathcal{C}} 3\right) \succ^{\mathcal{C}}\left(13 \sim^{\mathcal{C}} 23\right) \succ^{\mathcal{C}} 12 \succ^{\mathcal{C}} \emptyset \succ^{\mathcal{C}} 123 .
$$

The new scores are: Score $\frac{\succ_{K}^{\mathcal{c}}}{{ }^{c}}(1)=1, \operatorname{Score}_{\bar{K}}^{\succ^{\mathcal{c}}}(2)=1$, Score $\overline{\bar{K}}_{\bar{C}}^{\succ^{c}}(3)=1$. So, individual 1 now gets the first position.

Remark 1. For $n=2$, the Copeland-like solution and the KS-like solution coincide with the CP-majority relation, hence these solutions are not manipulable for $n=2$.

\subsection{Ordinal Banzhaf}

In the same spirit of the Banzhaf index [Banzhaf III, 1964], the ordinal Banzhaf solution is based on counting the number of positive and negative ordinal marginal contributions.

Definition 5 (Ordinal marginal contribution [Khani et al., 2019]). Let $\succeq \in \mathcal{T}(\mathcal{P})$. The ordinal marginal contribution $m_{i}^{S}(\succeq)$ of player $i$ w.r.t. coalition $S, i \notin S$, in power relation $\succeq$ is defined as:

$$
m_{i}^{S}(\succeq)=\left\{\begin{aligned}
1 & \text { if } \quad S \cup\{i\} \succ S, \\
-1 & \text { if } S \succ S \cup\{i\}, \\
0 & \text { otherwise. }
\end{aligned}\right.
$$

Definition 6 (Ordinal Banzhaf relation). Let $\succeq \in \mathcal{T}(\mathcal{P})$. The ordinal Banzhaf relation is the binary relation $R_{\bar{B} \text { anz }}^{\succ}$ such that for all $i, j \in N$ :

$$
i R_{\bar{B} \text { anz }}^{\succ} j \Leftrightarrow \operatorname{Score}_{\bar{B} \text { anz }}^{\succ}(i) \geq \operatorname{Score} e_{\bar{B} \text { anz }}^{\succ}(j),
$$

where $\operatorname{Score} e_{\bar{B} \text { anz }}^{\succ}(i)=u_{i}^{+, \succeq}-u_{i}^{-, \succeq}$ and $u_{i}^{+, \succeq}\left(u_{i}^{-, \succeq}\right)$ is defined as the number of coalitions $S$ with $i \notin S$ such that $m_{i}^{S}(\succeq)=1\left(m_{i}^{S}(\succeq)=-1\right)$.

Theorem 3. The Ordinal-Banzhaf solution is manipulable.

Proof. See Example 6 for an instance of manipulation.
Example 6. Consider $\succeq: 13 \succ 1 \succ 12 \succ 23 \succ 2 \succ$ $3 \succ 123 \succ \emptyset$. Then, $1 I_{\bar{B} \text { anz }}^{\succ} 3 P_{\bar{B} \text { anz }}^{\succ} 2$ since Score $e_{\bar{B} \text { anz }}^{\succ}(1)=$ $2, \operatorname{Score}_{\bar{B} \text { anz }}^{\succ}(2)=0$, Score $_{\bar{B} \text { anz }}^{\succ}(3)=2$. However, if 2 undermines the cooperation with 1 and $3(\mathcal{C}=\{12,23\})$ : $13 \succ^{\mathcal{C}} 1 \succ^{\mathcal{C}} 2 \succ^{\mathcal{C}} 23 \succ^{\mathcal{C}} 3 \succ^{\mathcal{C}} 12 \succ^{\mathcal{C}} 123 \succ^{\mathcal{C}} \emptyset$. Then the three individuals would have a null Banzhaf score and would be ranked equally.

\subsection{Lexicographic Excellence Solution}

The idea of lexicographic excellence is based on the lexicographic comparison of the frequency of individuals within equivalence classes, and taking care to reward individuals within the most excellent ones. Given the power relation $\succeq$ and its associated quotient ranking $\Sigma_{1} \succ \Sigma_{2} \succ \Sigma_{3} \succ \cdots \succ$ $\Sigma_{m}$, we denote by $i_{k}$ the number of sets in $\Sigma_{k}$ containing $i$ :

$$
i_{k}=\left|\left\{S \in \Sigma_{k}: i \in S\right\}\right|
$$

for $k=1, \ldots, l$. Now, let $\theta \succeq(i)$ be the $l$-dimensional vector $\theta^{\succeq}(i)=\left(i_{1}, \ldots, i_{l}\right)$ associated to $\succeq$. Consider the lexicographic order $\geq_{L}$ among vectors $\mathbf{i}$ and $\mathbf{j}: \mathbf{i} \geq_{L} \mathbf{j}$ if either $\mathbf{i}=$ $\mathbf{j}$ or there exists $t: i_{r}=j_{r}, r=1, \ldots, t-1$, and $i_{t}>j_{t}$.

Definition 7 (Lexicographic-excellence solution [Bernardi $e t$ al., 2019]). Let $\succeq \in \mathcal{T}(\mathcal{P})$. The lexicographic excellence (lexcel) relation is the binary relation $R_{\text {lexcel }}^{\succ}$ such that for all $i, j \in N$ :

$$
i R_{\text {lexcel }}^{\succeq} j \Leftrightarrow \theta^{\succeq}(i) \quad \geq_{L} \quad \theta^{\succeq}(j) .
$$

Example 7. Consider the power relation of Example 2. We have $\theta^{\succeq}(1)=(0,2,1)$ (since 1 is twice in $\Sigma_{2}$ and once in $\left.\Sigma_{3}\right), \theta^{\succeq}(2)=(1,2,0), \theta^{\succeq}(3)=(1,1,1)$, which yields the following lexcel ranking: $2 P_{\text {lexcel }}^{\succ} 3 P_{\text {lexcel }}^{\succeq} 1$.

Theorem 4. The lexcel solution is not manipulable.

Proof. Let $\succeq \in \mathcal{T}(\mathcal{P})$ be a power relation on $\mathcal{P} \subseteq 2^{N}$ with the associated quotient order $\succ$ :

$$
\Sigma_{1} \succ \Sigma_{2} \succ \cdots \succ \Sigma_{j} \succ \cdots \succ \Sigma_{k} \cdots \succ \Sigma_{m},
$$

and let $\mathcal{C}=\left\{S_{1}, S_{2}, \ldots, S_{l}\right\} \subseteq \mathcal{P}$ and $i \in N$ be such that $i \in \bigcap_{S \in \mathcal{C}} S$ (wlog, assume $S_{1} \succeq S_{2} \succeq \ldots \succeq S_{l}$ ).

Suppose there exists a manipulation $\succeq^{\mathcal{C}}$ of $\succeq$ by $i$ such that $p_{i}\left(R^{\succeq^{\mathcal{C}}}\right) \geq p_{i}\left(R^{\succeq}\right)$. Then there must be some $k \in N \backslash\{i\}$ such that

$$
k R_{\text {lexcel }}^{\succ} i \text { and } i R_{\text {lexcel }}^{\succ^{\mathcal{C}}} k .
$$

First, notice that there exists some coalition $S \in \mathcal{C}$ such that $k \notin S$ (otherwise, if $i, k \in \bigcap_{S \in \mathcal{C}} S$, the manipulation would have no impact on the relative comparison of $i$ and $k$, since in this case $\left.\theta^{\succeq}(i) \geq_{L} \theta^{\succeq}(k) \Leftrightarrow \theta^{\succeq^{c}}(i) \geq_{L} \theta^{\succeq^{\mathcal{C}}}(k)\right)$.

Now let $S^{*} \in \mathcal{C}$ be a coalition not containing $k$ with the smallest index in $\mathcal{C}$, and let $j\left(S^{*}\right) \in\{1, \ldots, m\}$ be such that $S^{*} \in \Sigma_{j\left(S^{*}\right)}$. Since $k R_{\text {lexcel }}^{\succ} i$, we distinguish two cases:

i) $k I_{\text {lexcel }}^{\succeq}(k$ and $i$ are indifferent in $\succeq$ according to the lexcel relation). Then, by Definition 7, $\theta \frac{\succ_{v}^{c}}{}(k)=$ $\theta_{\bar{v}}^{\succ}(k)=\theta_{\frac{\zeta}{v}}^{\succeq}(i)=\theta_{\frac{v^{c}}{c}}(i)$ for all $v<j\left(S^{*}\right)$, while $\theta_{j\left(S^{*}\right)}^{\succ^{\mathcal{C}}}(k)>\theta_{j\left(S^{*}\right)}^{\succ^{\mathcal{C}}}(i)$. So, $k P_{\text {lexcel }}^{\succ^{\mathcal{C}}} i$; 
ii) $k P_{\text {lexcel }}^{\succeq} i$ ( $k$ is strictly stronger than $i$ in $\succeq$ according to the lexcel relation). So, let $t$ be the smallest index such that $\theta_{t}^{\succeq}(k)>\theta_{t}^{\succeq}(i)$. Moreover, let $q=\min \left(t, j\left(S^{*}\right)\right)$.

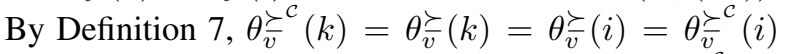
for all $v<q$ and $\theta_{\bar{q}}^{\succ^{\mathcal{C}}}(k)>\theta_{\bar{q}}^{\succ^{\mathcal{C}}}(i)$. So, again, $k P_{\text {lexcel }}^{\succeq^{\mathcal{C}}} i$. In both cases we get a contradiction with the fact that $i R_{\text {lexcel }}^{\succ^{\mathcal{C}}} k$ by relation (4). Since $\mathcal{C}$ is an arbitrary collection of coalitions in $\mathcal{P}$, we have proved that the lexcel solution is not manipulable.

A drawback of the lexcel solution is that it makes use of a limited amount of information, giving a quasi-dictatorial power to the best ranked coalitions. On the other hand, Copeland-like and KS-like rules are based on the intuitive idea of ceteris paribus comparisons and have the advantage of choosing the Condorcet winner (with respect to the $\mathrm{CP}$ majority relation) when it exists. The Ordinal Banzhaf rule, based on the intuitive notion of ordinal marginal contribution of individuals, also offers another interesting interpretation in terms of fairness. In order to further study the effective impact of manipulation on these three appealing solutions, in Section 4 we analyse the computational complexity of manipulation and in Section 5 we introduce some simulations on several numerical instances.

\section{Computational Complexity of Manipulation}

As we have seen in the previous section, the Copeland-like solution, the KS-like solution, and the Ordinal Banzhaf solution are manipulable. We strengthen these results in this section by showing that, for each of these social ranking solutions, determining whether an individual can manipulate or not is an NP-hard problem. Let us state the problem precisely. As an instance, we have a set $N=\{1, \ldots, n\}$ of individuals with a manipulator $t \in N$, a set $\mathcal{P} \subseteq 2^{N}$, and a power relation $\succeq$ on $\mathcal{P}$. The question is to determine whether a given solution is manipulable by $t$ on $\mathcal{P}$, as defined in Definition 1 .

Theorem 5. For the Copeland-like solution, the KS-like solution, and the Ordinal Banzhaf solution, the manipulation problem is NP-hard.

Proof. Due to lack of space, we only present the proof for the Ordinal Banzhaf solution. We build the following instance of the manipulation problem under the Ordinal Banzhaf solution. First, let us consider the following individuals:

- we associate to each edge $e_{i} \in E$ an individual that we call $e_{i}$ as well (for convenience), and to each vertex $v$ an individual that we call $v$ as well (for convenience);

- two other individuals: $t$ (the manipulator), and $\alpha$.

For each vertex $v$, let us call $\mathcal{P}_{v}$ the set containing the subsets of individuals $\{v, \alpha, t\},\{v, t\}$ and all subsets $\left\{v, e_{i}, \alpha, t\right\}$ for each edge $e_{i}$ incident to $v$. $\mathcal{P}_{v}$ is ordered as follows in $\succeq$ : $\{v, \alpha, t\}$ is the first one (strictly preferred to any other sets in $\left.\mathcal{P}_{v}\right),\{v, t\}$ is the last one, and all $\left\{v, e_{i}, \alpha, t\right\}$ are equivalent, ranked between $\{v, \alpha, t\}$ and $\{v, t\}$.

Note that each set in $\mathcal{P}_{v}$ contains $v$, so for the scores the relative positions of 2 sets in $\mathcal{P}_{v}$ and $\mathcal{P}_{v^{\prime}}$ do not matter; we do not specify it.
The contribution of these sets $\mathcal{P}_{v}$ to the scores are: $+|V|$ for $\alpha$ (due to $\{v, \alpha, t\} \succ\{v, t\}$ for each vertex/individual $v$ ), -2 for each object $e_{i}$ (due to $\{v, \alpha, t\} \succ\left\{v, e_{i}, \alpha, t\right\}$, for each of the two extremities of edge $e_{i}$ ), and 0 for $t$ (each set contains $t$ ).

The idea of the reduction is that, in order to manipulate, $t$ has to become first (defeating $\alpha$ ). To do this she shall put $\{v, \alpha, t\}$ below $\{v, t\}$ in some $\mathcal{P}_{v}$. But doing this, the score of the edges incident to $v$ increases. $t$ cannot do this for the two extremities of an edge, otherwise $e_{i}$ defeats him.

To make this true, we need to add dummy individuals to adjust the initial scores of $\alpha$ and $t$. For the score of $\alpha$, we add $\lambda=2 k-|V|$ individuals $b_{1}, \ldots, b_{\lambda}$. For each $b_{i}$, we order $\left\{b_{i}, \alpha\right\} \succ\left\{b_{i}\right\}$. This gives an extra score of $\lambda=2 k-|V|$ to $\alpha$, while $b_{i}$ has score 0 . Finally, we add an object $\gamma$, and order $\{\gamma, t\} \succ\{\gamma\}$, giving an extra score of 1 to the manipulator $t$.

Note that as previously we do not need to further specify $\succeq$, since the relative positions of sets containing different $b_{i}$, and/or $\gamma$, and/or in different $\mathcal{P}_{v}$, does not matter with respect to the scores (there is no other set inclusion).

To sum up, we have $|E|+|V|+2+(2 k-|V|)+1$ individuals: each individual $e_{i}$ has score -2 , individual $t$ has score 1 , and $\alpha$ has score $|V|+2 k-|V|=2 k$. All dummy objects and objects $v$ have score 0 . Note that the size of $\mathcal{P}$ is polynomial in the size of $G$.

We claim that $t$ can manipulate if and only if there is an independent set of size $k$ in $G$.

Suppose that there is an independent set $S$ of size $k$ in $G$. Then consider the manipulation where, for each $v$ in $S, t$ puts $\left\{v, e_{i}^{j}, \alpha, t\right\}$ down to the last position in $\mathcal{P}_{v}$. Then the score of $\alpha$ decreases by $2 k$ and becomes 0 . The score of $e_{i}$ is modified in at most one $\mathcal{P}_{v}$, since $S$ is an independent set, so it is at most 0 . The score of $t$ is still 1 , and $t$ manipulated the election.

Conversely, suppose that $t$ can manipulate. Note that $t$ cannot increase her own score, so she must make the score of $\alpha$ at most 1 . This means that she has to put $\{v, \alpha, t\}$ in the last position in at least $k$ sets $\mathcal{P}_{v}$. Let $S$ be the corresponding set of vertices. If $S$ contains both extremities of one edge $e_{i}$, then the score of $e_{i}$ becomes +2 , and $t$ is not better of. So, in order to manipulate, the set $S$ must be an independent set, and it is of size at least $k$.

\section{Simulations}

Inspired by previous works in voting theory [Chamberlin, 1985], we study to what extend the three rules are manipulable. In other terms, based on computer simulations of various power relations, we estimate the probability that a manipulation occurs and we analyse the vulnerability of each solution to manipulation. We only consider situations with a single manipulator and power relations on the whole set $2^{N}$. In order to perform our simulations we need to find a manipulation strategy for each rule under the assumption that the manipulator has a complete knowledge about the power relation.

To find a manipulation, we set up an integer linear programming (ILP) formulation of the problem (not detailed here due to lack of space), the variables of which represent the ranking after manipulation. This ILP is efficient enough 


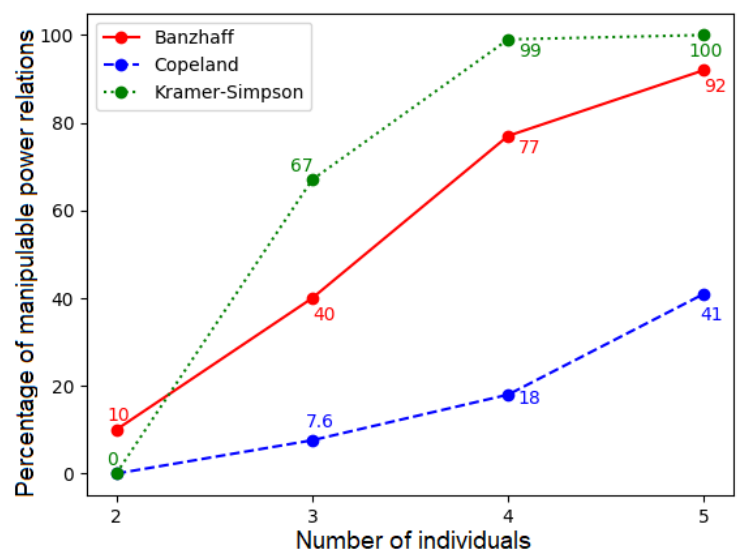

Figure 1: Percentage of manipulable cases as a function of $n=|N|$.

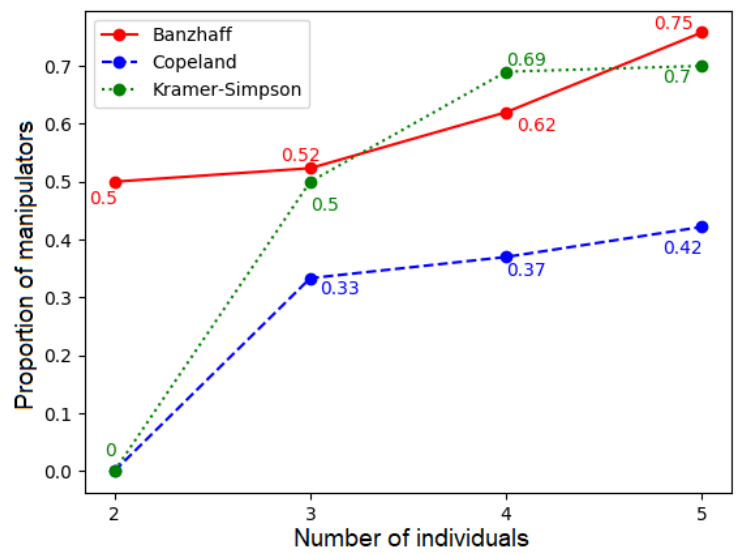

Figure 2: Proportion of manipulators as a function of $n=|N|$.

for small values of $n$, and we perform our simulation on total power relations over $2^{N}$ up to $n=5$, i.e., power relations on up to $2^{5}=32$ coalitions. The data generation is done using Monte Carlo methods, following uniform (impartial culture) model, which assumes that all power relations over coalitions are equally likely to occur. For each number of individuals $n$, we generated 1000 random total power relations.

The proportion of manipulable cases. A power relation is manipulable if there exists at least one individual who can manipulate it. The probability of having a manipulable power relation increases rapidly with the number of individuals for the three solutions, especially for the KS relation for which it reaches $99.6 \%$ for $n=4$ and $100 \%$ for $n=5$. However, for Copeland it reaches $41 \%$ and $92 \%$ for Banzhaf (see Figure 1).

Number of possible manipulators. We look at the number of possible manipulators for each manipulable case. The results are shown in Figure 2. The proportion of manipulators grows with the number of individuals. We note also that for Copeland solution, there are on average less possible manipulators for each power relation, and thus has a lower probability of being actually manipulated by one of them.

Manipulating to be the best ranked. We analyse in the following the probability of becoming the best ranked one

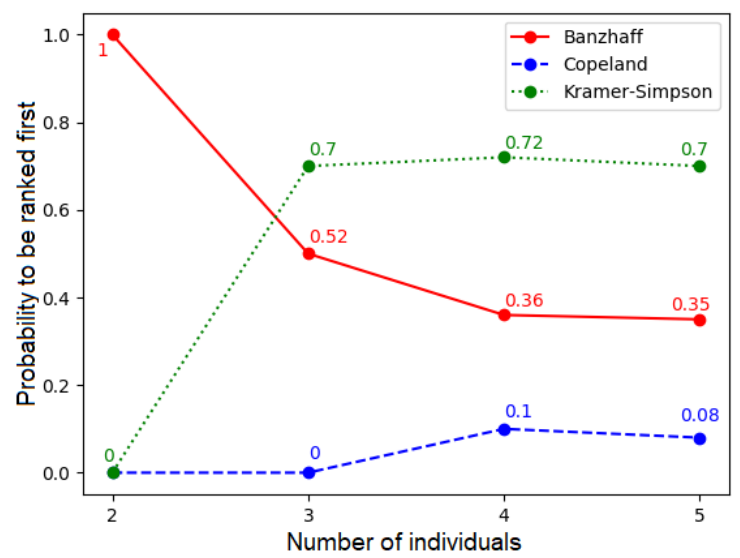

Figure 3: Probability to be ranked first as a function of $n=|N|$.

\begin{tabular}{|l|c|c|c|}
\hline manipulable by & $N=3$ & $N=4$ & $N=5$ \\
\hline Banz. +Cop. +KS & 7.4 & 16.4 & 33.1 \\
\hline Banz. +Cop. & 0 & 0 & 0 \\
\hline Banz. +KS & 29.6 & 61.2 & 56.2 \\
\hline Banz. & 1 & 0 & 0 \\
\hline Cop. +KS & 0.2 & 0.4 & 4.7 \\
\hline Cop. & 0 & 0 & 0 \\
\hline KS & 28.7 & 22.0 & 6.0 \\
\hline none & 33.1 & 0 & 0 \\
\hline
\end{tabular}

Table 1: Percentage of manipulable power relations.

(ties are possible) thanks to a manipulation (see Figure 3).

Cross-simulation. We end our analysis by a cross simulation where for a given power relation we analyze the manipulability with respect to each social ranking rule (Table 1). Table 1 is coherent with our previous results (see Figure 1). Based on the results of simulations, it seems that if a power relation is manipulable by the Copeland-like solution, it is also by the KS-like solution. The most common case is to be manipulable by ordinal the Ordinal Banzhaf solution and the KS-like solution. For these reasons, our conjecture (suggested by the experimental results) is that the Copeland-like solution is not manipulable alone.

\section{Conclusion}

We have studied the problem of manipulating social ranking solutions. We have shown that lexcel is not manipulable and the manipulation of three other rules is NP-hard. Using simulation, we have remarked that Copeland-like is more resistant to manipulation than the Ordinal Banzhaf solution and the KS-like solution. Our study opens the way for many future works. We quote some of them : An axiomatic characterization of SRC rules taking into account strategy-proofness (like the one of Gibbard and Satterhwaite ([Gibbard, 1973] and [Satterthwaite, 1975])), analysis of the impact on the manipulability of some domain restrictions, study of coalitional manipulation or of simultaneous manipulation (game theoretical issues). 


\section{References}

[Aziz et al., 2011] Haris Aziz, Yoram Bachrach, Edith Elkind, and Mike Paterson. False-name manipulations in weighted voting games. J. Artif. Intell. Res., 40:57-93, 2011.

[Bachrach and Rosenschein, 2009] Yoram Bachrach and Jeffrey S Rosenschein. Power in threshold network flow games. Autonomous Agents and Multi-Agent Systems, 18(1):106, 2009.

[Banzhaf III, 1964] John F Banzhaf III. Weighted voting doesn't work: A mathematical analysis. Rutgers L. Rev., 19:317, 1964.

[Bernardi et al., 2019] Giulia Bernardi, Roberto Lucchetti, and Stefano Moretti. Ranking objects from a preference relation over their subsets. Social Choice and Welfare, 52(4):589-606, 2019.

[Bilbao et al., 2002] Jesús Mario Bilbao, Julio R Fernandez, Nieves Jiménez, and Jorge J Lopez. Voting power in the european union enlargement. European Journal of Operational Research, 143(1):181-196, 2002.

[Chamberlin, 1985] John R. Chamberlin. An investigation into the relative manipulability of four voting systems. $\mathrm{Be}$ havioral Science, 30(4):195-203, 1985.

[Chiaramonte et al., 2020] Alessandro Chiaramonte, Lorenzo De Sio, and Vincenzo Emanuele. Salvini's success and the collapse of the five-star movement: The european elections of 2019. Contemporary Italian Politics, pages 1-15, 2020.

[Copeland, 1951] Arthur H. Copeland. A reasonable social welfare function. Technical report, mimeo, 1951. University of Michigan, 1951.

[Deng and Papadimitriou, 1994] Xiaotie Deng and Christos H Papadimitriou. On the complexity of cooperative solution concepts. Mathematics of Operations Research, 19(2):257-266, 1994.

[Faliszewski and Hemaspaandra, 2009] Piotr Faliszewski and Lane Hemaspaandra. The complexity of powerindex comparison. Theoretical Computer Science, 410(1):101-107, 2009.

[Fayard and Öztürk, 2018] Nicolas Fayard and Meltem Öztürk. Ordinal Social ranking: simulation for CPmajority rule. In Multiple Criteria Decision Aid to Preference Learning, DA2PL 2018, Poznan, Poland, November 2018.

[Felsenthal et al., 1998] Dan S Felsenthal, Moshé Machover, et al. The measurement of voting power. Books, 1998.

[Ferrara, 2004] Federico Ferrara. Electoral coordination and the strategic desertion of strong parties in compensatory mixed systems with negative vote transfers. Electoral studies, 23(3):391-413, 2004.

[Flores-Szwagrzak and Treibich, 2020] Karol FloresSzwagrzak and Rafael Treibich. Teamwork and individual productivity. Management Science, 2020.
[Gibbard, 1973] Allan Gibbard. Manipulation of voting schemes. Econometrica, 41:587-601, 1973.

[Haret et al., 2018] Adrian Haret, Hossein Khani, Stefano Moretti, and Meltem Öztürk. Ceteris paribus majority for social ranking. In Proceedings of the 27th International Joint Conference on Artificial Intelligence, IJCAI 2018, pages 303-309, 2018.

[Holler and Packel, 1983] Manfred J Holler and Edward W Packel. Power, luck and the right index. Zeitschrift für Nationalökonomie, 43(1):21-29, 1983.

[Khani et al., 2019] Hossein Khani, Stefano Moretti, and Meltem Öztürk. An ordinal banzhaf index for social ranking. In Proceedings of the 27th International Joint Conference on Artificial Intelligence, IJCAI 2019, 2019.

[Kramer, 1977] Gerald H. Kramer. A dynamical model of political equilibrium. Journal of Economic Theory, 16(2):310-334, December 1977.

[Laruelle and Valenciano, 2001] Annick Laruelle and Federico Valenciano. Shapley-shubik and banzhaf indices revisited. Mathematics of operations research, 26(1):89104, 2001.

[Laruelle and Valenciano, 2005] Annick Laruelle and Federico Valenciano. A critical reappraisal of some voting power paradoxes. Public Choice, 125(1-2):17-41, 2005.

[Matsui and Matsui, 2000] Tomomi Matsui and Yasuko Matsui. A survey of algorithms for calculating power indices of weighted majority games. Journal of the Operations Research Society of Japan, 43(1):71-86, 2000.

[Moretti and Öztürk, 2017] Stefano Moretti and Meltem Öztürk. Some axiomatic and algorithmic perspectives on the social ranking problem. In Algorithmic Decision Theory - 5th International Conference, ADT 2017, Luxembourg, Luxembourg, October 25-27, 2017, Proceedings, pages 166-181, 2017.

[Moretti and Patrone, 2008] Stefano Moretti and Fioravante Patrone. Transversality of the shapley value. Top, 16(1):1, 2008.

[Moretti, 2015] Stefano Moretti. An axiomatic approach to social ranking under coalitional power relations. Homo Oeconomicus, 32(2):183-208, 2015.

[Satterthwaite, 1975] Mark Allen Satterthwaite. Strategyproofness and arrow's conditions: Existence and correspondence theorems for voting procedures and social welfare functions. Journal of Economic Theory, 10(2):187$217,1975$.

[Shapley, 1953] Lloyd S Shapley. A value for n-person games. Contributions to the Theory of Games, 2(28):307317, 1953.

[Simpson, 1969] Paul B. Simpson. On Defining Areas of Voter Choice: Professor Tullock on Stable Voting. The Quarterly Journal of Economics, 83(3):478-490, 081969.

[Zuckerman et al., 2012] Michael Zuckerman, Piotr Faliszewski, Yoram Bachrach, and Edith Elkind. Manipulating the quota in weighted voting games. Artif. Intell., 180-181:1-19, 2012. 